\title{
RESPONSE OF BARLEY AND VETCH MIXTURE TO PHOSPHORUS FERTILIZER LEVELS UNDER RAINFED CONDITIONS AT RAS EL-HEKMA, MATROUH, EGYPT Mohamed, N. M. ${ }^{1}$ and R. M. Aly ${ }^{2}$ \\ ${ }^{1}$ Sustainable Development Center for Matrouh Resources, Desert Research Center, Egypt . \\ ${ }^{2}$ Agronomy Department., Fuclty of Agriculture., Zagazig Univ., Egypt. \\ E. mail : naem20042001@yahoo.com
}

\begin{abstract}
Two field experiments were conducted at South of Ras El-Hekma (56 km east of Marsa Matrouh city) under rainfed conditions during 2005/ 2006 and 2006/ 2007 winter seasons, to show the response of pure stands of barley (Hordeum vulgare L.), vetch (Vicia sativa L.) and their mixture (75\% barley and $25 \%$ vetch) to P-fertilizer levels $\left(0,7.5,15\right.$ and $22.5 \mathrm{Kg} \mathrm{P} 2 \mathrm{O}_{5} /$ fad) to study the effect of cropping systems and P-fertilization on grain and straw yields, as well as, field stubble and water use efficiency.

The obtained results scribed that, barley- vetch mixture $(75 \%$ barley $+25 \%$ vetch) at different growth stages; i.e., booting, $50 \%$ heading and physiological stages, was superior to mono-cropping system of barley and vetch in fresh forage, dry matter and field stubble yields, as well as, crude protein yield in both seasons. Also, at harvesting time; i.e., full maturity stage, B-V mixture gave the highest grain and straw yields with higher total crude protein yield. Mixed cropping system was efficiently in water-use compared to pure stands of barley and vetch. The land equivalent ratio (LER) was more than one for mixed cropping system, which also, led to the highest LER in respect to dry matter yield, crude protein yield at different growth stages and harvesting at full maturity. Applying of higher P-fertilizer level; i.e., $22.5 \mathrm{Kg} \mathrm{P}_{2} \mathrm{O}_{5} / \mathrm{fad}$ gave the highest of studied traits. Economically, barley-vetch mixture with $\mathrm{P}$-fertilizer level of $22.5 \mathrm{Kg} \mathrm{P}_{2} \mathrm{O}_{5} /$ fad gave the highest values of gross and net returns, while, $\mathrm{B}-\mathrm{V}$ mixture with application of $15 \mathrm{Kg} \mathrm{P}_{2} \mathrm{O}_{5} / \mathrm{fad}$ achieved the highest values of average rate of return (ARR). Ultimately, these results ascertained the importance of entering some of contingent forage crops, such as vetch to associate with barley in mixedcropping system particularly, with P-fertilization, also to improve the crop/ livestock farming system, which might be contribute in sustainable development of rainfed conditions at Ras El-Hekma, Matrouh, Northwestern coast of Egypt.
\end{abstract}

Keywords: Mixed cropping system, mono-cropping, land equivalent ratio, vetch, $\mathrm{P}$ fertilization, water use efficiency, barley-vetch mixture, growth stages.

\section{INTRODUCTION}

Livestock in particular small ruminants is an important component in the Northwestern coastal (NWC) zone of Egypt which characterized by fragile dry farming systems under rainfed conditions. The importance of livestock in this area might be due to the fact that the main income source $(65 \%)$ for about $75-80 \%$ of Bedouin farmers is resulted from livestock flocks (MRMP, 2002). However, depending on grazing of the natural vegetation cover to provide these flocks with its feed needs become inconvenient in the case of continuous the deterioration of natural rangelands due to heavy grazing and 
climate changes; i.e., drought, heat stress and fluctuation of rainfall. Hence, tame pasture culture establishment is an urgent request for sustainable development in such environmental conditions.

It is conspicuous that the continuous cultivation of barley, as a dominant and adapted crop in the NWC area, has been adverse effects on its yield potentiality and sustainable production of palatable native legume plants. Accordingly, long-term mono-cropping system of cereals will be providing animals with unbalanced diet. In addition to, exposure the soil surface to wind erosion due to continuous plowing for sole cereals every year without natural plant cover to protect the soil surface. Meantime, barley has sufficient amount of carbohydrates, but, it's deficient in protein content which is necessary for animal productivity. Based on these points of view, it is importance to introduce some agronomic practices such as a suitable cropping system to raising the yielding capacity of barley and to improve forage quality. Mixed cropping of legumes and cereals is uncommon in the low rainfall areas such NWC of Egypt. But, in many parts of the world, farmers use cereals and legumes in mixtures to improve nutritive value and productivity of pasture besides taking advantages of the enhanced biological $\mathrm{N}_{2}$ fixation. Vetch (Vicia sativa L. and Vicia monantha L.) is an important forage crop under rainfed or irrigated conditions in the Middle East. In Cyprus, vetch is widely used for forage production in a mixed cropping system with oat (Papastylianou and Danso, 1989 \& 1991). Likewise, in Syria, farmers prefer forage production of vetch with barley over sole of both and these mixtures can be considered as a direct grazing feed for animal during winter and early spring. In the NWC of Egypt, Moselhy and El-Hakeem (2001) concluded that barley-vetch mixture with 75 and $25 \%$ of their seeds, respectively secured the highest dry forage yield and achieved the greatest advantages in carbohydrate and protein yields as compared with monocropping of barley or vetch. In Iran, Mohsenabadi et al. (2008) reported the intercropping vetch and barley maximized productivity and crude protein yield, as well as, highest land equivalent ratio (LER) and water use efficiency (WUE) for grain production. In comparison among pure stands of cereals and their mixtures with legumes fodder crops, different studies cleared that benefits of mixed cropping may arise from increased $\mathrm{N}$ and dry matter production Fujita et al. (1990), greater $\mathrm{N}_{2}$ fixation efficiency for subsequent crop (Izaurralde et al., 1992), and land use efficiency (Offori and Stem., 1987 and Kurdali et al., 1996). Also, the mixed cropping of vetch with oat, barley and wheat may affect the growth rate of the individual species in mixtures, as well, as, the forage yield and quality (Lithourgidis et al., 2006). Recently, under rainfed conditions at Pakistan, Ansar et al. (2010) found that oat-vetch mixture performed better under rainfed conditions in terms of green and dry matter yield and attained higher crude protein content than their respective pure stands. Vetches as a legume forage crop not only improve soil fertility through biological $\mathrm{N}$ fixation but also have excellent nutritive value and also, grown as cover crop to protect the soil form erosion. Qamar et al. (1999) found that the dry matter yield and quality of forage mixture of vetch and barley are highly promising being the potential option to farmers in the rainfed areas. Phosphorus application significantly affects the forage yield and quality 
of vetch (Turk and Tawaha, 2001; Karadag and Buyukburc, 2001 and Gurman et al., 2006).

Therefore, the present study objectives was aimed to show the forage yield potential of barley and vetch pure stands and their mixture as affected by phosphorus fertilizer levels under rainfed conditions at Ras El-Hekma, Matrouh, NWC of Egypt.

\section{MATERIALS AND METHODS}

Two field experiments were conducted under rainfed conditions at Ras El-Hekma (10 km inland), $56 \mathrm{~km}$ east Marsa Matrouh, NWC of Egypt, during 2005/ 2006 and 2006/ 2007 winter growing seasons, to show the response of pure stands of barley (Hordeum vulgare L.), vetch (Vicia sativa L.) and their mixture ( $75 \%$ barley and $25 \%$ vetch) to phosphorus fertilizer levels; i.e., 0 , $7.5,15$ and $22.5 \mathrm{Kg} \mathrm{P}_{2} \mathrm{O}_{5} / \mathrm{fad}$. Also, to find out the effect of cropping systems and P-fertilizer levels on grain and straw yields, as well as, field stubble and water use efficiency.

Each experiment included 12 treatments, which were arranged in a split plot design in four replications. Three cropping systems treatments were allocated in the main plots, while four P-fertilizer levels were distributed randomly in the sub-plots. Every sub-plot area was $100 \mathrm{~m}^{2}$. Pure stand of barley (Giza $126 \mathrm{cv}$.) at seeding rate of $30 \mathrm{Kg} / \mathrm{fad}$, pure stand of vetch (Vicia sativa L.) at seeding rate of $40 \mathrm{Kg} / \mathrm{fad}$ and barley-vetch mixture at a seeding rates equivalent to $75 \%$ and $25 \%$ of pure stands of barley and vetch, respectively were sown after the first effective rainfall precipitation on November 12 and 15 in the first and second seasons, in respective order. Seeds of vetch were soaked in fresh water for $48 \mathrm{hr}$, in order to raise their germination percentage from $45-50 \%$ to $95 \%$. The tried treatments of phosphorous fertilizer levels were applied in form of calcium superphosphate $\left(15.5 \% \mathrm{P}_{2} \mathrm{O}_{5}\right)$ at sowing time with one dose. All agronomic practices were applied as recommended for rainfed agriculture.

The soil of the experimental site was sandy-loam in texture. Soil chemical analysis indicated the presence of 0.014-0.017\% available nitrogen, 13-19 ppm phosphorous and high $\mathrm{CaC}_{3}$ content (25-28 \%). The $\mathrm{EC}$ of the soil ranged between 0.82 to $0.86 \mathrm{dsm}$ and $\mathrm{pH}$ was 7.65 ( Lab of Desert Research Center at Matrouh).

Monthly rainfall precipitation (mm) during the 2005/ 2006 and 2006/ 2007 seasons as presented in Table (1).

Table 1: Monthly rainfall precipitation $(\mathrm{mm})$ during the 2005/ 2006 and 2006/ 2007 seasons.

\begin{tabular}{|c|c|c|c|c|c|c|c|}
\hline Season & Oct. & Nov. & Dec. & Jan. & Feb. & March & Total $(\mathbf{m m})$ \\
\hline $2005 / 2006$ & 7.6 & 32.9 & 34.2 & 33.5 & 5.0 & 0.0 & 113.2 \\
\hline $2006 / 2007$ & 0.0 & 33.5 & 22.6 & 31.0 & 33.0 & 20.0 & 140.1 \\
\hline
\end{tabular}


To determine fresh and dry forage yields, as well as, forage quality at different growth stages, single forage harvest was taken per each growth stage i.e. 105, 125 and 125 days after sowing, which coincided to booting, 50 $\%$ heading and physiological maturity for barley, in respective order. At each growth stage, plants in $15 \mathrm{~m}^{2}$ of each sub-plot were harvested then weighted and sub-samples were oven dried at $70^{\circ} \mathrm{C}$ to estimate dry mater yield and forage quality. Plants from the mixed cropping system treatment were separated into component crops (barley and vetch). The rest area of each sub-plot was used to determine grain and straw yields at full maturity stage of barley plants. At the end of the season, field stubble which reissued form each harvest time was also determined.

Land equivalent ratio (LER) was used for estimating the efficiency of mixed cropping relative to mono-cropping systems and was calculated according to the method of Mead and Willey (1980), i.e. LER $=($ Yij / Yii $)+$ $\left(Y j i\right.$ / Yjj). Where, $Y$ is the yield per area unit, Yii and $Y_{j j}$ are pure stand yields for the component crops I and j. Yij and Yji are mixed crop yields. At harvest in the end of season (full maturity stage), total Scandinavian feed units (TSFUs) were estimated (total sum of SFUs for grain, straw and field stubble yields) where, one $\mathrm{Kg}$ of barley grain equals one SFU, while one $\mathrm{Kg}$ of dry matter equals 0.45 SFU (Le Houerou, 1986). Also, water use efficiency (WUE) for grain production and Scandinavian feed units yield was calculated according to Le Houerou (1994) as follows:

WUE $E_{G P}=\mathrm{GY}(\mathrm{kg} / \mathrm{fad}) \div \mathrm{TR}(\mathrm{mm} /$ season $)$.

WUESFUs $=$ TSFUs (SFU/ fad) $\div$ TR $(\mathrm{mm} /$ season) .

Where, WUE $E_{G P}$ is water use efficiency for grain production, WUESFUs is water use efficiency for total Scandinavian feed units, GY is grain yield (kg/ fad), TSFUs is total Scandinavian feed units/ fad and TR is the total rainfall $(\mathrm{mm})$ / season.

The collected data were statistically analyzed, according to (Snedecor and Cochran, 1990). Mean values were compared at $P<0.05$ using the Least Significant Difference (LSD) test. Economic analysis using partial budget was made according to Perrin, et al. (1983) as follows:

GR (LE) $=$ TSFUs $/$ fad $x$ price of unit.

$\mathrm{NR}(\mathrm{LE})=\mathrm{GR} / \mathrm{fad}-\mathrm{TC} / \mathrm{fad}$.

ARR $(\mathrm{LE})=\mathrm{NR} / \mathrm{fad} \div \mathrm{TC} / \mathrm{fad}$.

Where, GR is gross return (LE/ fad, TSFUs is total Scandinavian feed units/ fad, NR is net return (LE/ fad), TC is total costs (LE/ fad) and ARR is average rate of return (LE/ fad).

\section{RESLUTS AND DISCUSSION}

\section{Fresh and dry matter yields:}

Results related to the effect of cropping systems and P-fertilizer levels on fresh and dry matter yields at different growth stages are presented in Tables 2 and 3, respectively. It is clearly evident that barley-vetch mixture (75 $\%$ barley $+25 \%$ vetch) had the highest fresh and dry matter yields followed by pure stand of barley at different growth stages in both seasons. While, 
pure stand of vetch recorded the lowest values of the abovementioned two yields. This means that associated both crops used the limited environmental resources more efficiently than pure stands. Under rainfed conditions, Moselhy and El-Hakeem (2001) at NWC of Egypt, Ansar et al. (2010) at Pakistan and Nadeem et al (2010) at Iran, they reported that cereal-legume mixtures were more productive than mono-cropping systems.

Also, fresh and dry matter yields were increased consistently with each P-fertilizer increment up to the higher level tested here $\left(22.5 \mathrm{Kg} \mathrm{P}_{2} \mathrm{O}_{5} / \mathrm{fad}\right)$. This was typically the same at different growth stages in both seasons. Though soil of the experimental fields contains relatively higher available phosphorus, the response to P-fertilizer recorded herein could be explained through the high activity of calcium in phosphorus fixation in soil. Moreover, it is of noticeable that fresh and dry matter yields were increased with delayed forage harvest from booting and $50 \%$ heading to physiological maturity stage. Results in Table 3 showed that barley-vetch mixture produced higher dry matter (DM) yield than pure vetch by $68.3,40.6$ and $29.4 \%$ at booting, 50 $\%$ heading and physiological maturity stages, respectively in the first season. However, in the second season these percentage increases were 65.8, 39.9 and $29.1 \%$ in respective order. The higher increase percent in DM yield was obtained from harvesting at first early growth stage may be due to the efficient of associated barley in mixture to produce more number of tillers.

Table 2: Effect of cropping systems and P-fertilizer levels on fresh forage yield $(\mathrm{t} / \mathrm{fad})$ at booting, $50 \%$ heading and physiological maturity (P.M) stages of barley in both seasons

\begin{tabular}{|c|c|c|c|c|c|c|}
\hline \multirow{3}{*}{ Treatments } & \multicolumn{3}{|c|}{$2004 / 05$} & \multicolumn{3}{|c|}{$2005 / 06$} \\
\hline & Booting & Heading & P.M. & Booting & Heading & P.M. \\
\hline & stage & stage & stage & stage & stage & stage \\
\hline \multicolumn{7}{|c|}{ Cropping systems (C): } \\
\hline Pure barley & $2.070 \mathrm{~b}$ & $2.924 \mathrm{~b}$ & $3.549 \mathrm{~b}$ & $2.103 \mathrm{~b}$ & $2.983 \mathrm{~b}$ & $3.601 \mathrm{~b}$ \\
\hline Barley-Vetch & $2.355 \mathrm{a}$ & $3.150 \mathrm{a}$ & $3.709 \mathrm{a}$ & $2.392 \mathrm{a}$ & $3.210 \mathrm{a}$ & $3.753 \mathrm{a}$ \\
\hline Pure vetch & $1.398 \mathrm{c}$ & $2.243 c$ & $2.866 \mathrm{c}$ & $1.444 \mathrm{c}$ & $2.295 \mathrm{c}$ & $2.907 \mathrm{c}$ \\
\hline F. test $-5 \%$ & * & * & * & * & * & * \\
\hline \multicolumn{7}{|c|}{ P-fertilizer levels $(P)$ : } \\
\hline Zero & $1.610 \mathrm{~d}$ & $2.316 \mathrm{~d}$ & $2.998 \mathrm{~d}$ & $1.645 \mathrm{~d}$ & $2.377 \mathrm{~d}$ & $3.035 \mathrm{~d}$ \\
\hline $7.5 \mathrm{Kg} \mathrm{P}_{2} \mathrm{O}_{5} / \mathrm{fad}$ & $1.820 \mathrm{C}$ & $2.606 \mathrm{c}$ & $3.222 \mathrm{c}$ & $1.861 \mathrm{c}$ & $2.674 \mathrm{c}$ & $3.264 \mathrm{c}$ \\
\hline $15 \mathrm{Kg} \mathrm{P}_{2} \mathrm{O}_{5} / \mathrm{fad}$ & $2.065 \mathrm{~b}$ & $2.959 \mathrm{~b}$ & $3.532 \mathrm{~b}$ & $2.104 \mathrm{~b}$ & $3.015 \mathrm{~b}$ & $3.587 \mathrm{~b}$ \\
\hline $22.5 \mathrm{Kg} \mathrm{P}_{2} \mathrm{O}_{5} / \mathrm{fad}$ & $2.270 \mathrm{a}$ & $3.209 a$ & $3.748 \mathrm{a}$ & $2.309 \mathrm{a}$ & $3.251 \mathrm{a}$ & $3.795 \mathrm{a}$ \\
\hline F. test $-5 \%$ & * & * & * & * & * & * \\
\hline \multicolumn{7}{|l|}{ Interaction: } \\
\hline$C \times P$ & * & * & * & * & * & * \\
\hline
\end{tabular}

*: Significant at 0.05 level of probability. 
Table 3: Effect of cropping systems and P-fertilizer levels on dry matter yield $(\mathrm{t} / \mathrm{fad})$ at booting, $50 \%$ heading and physiological maturity (P.M) stages of barley during the two seasons.

\begin{tabular}{|c|c|c|c|c|c|c|}
\hline \multirow{3}{*}{ Treatments } & \multicolumn{3}{|c|}{$2004 / 05$} & \multicolumn{3}{|c|}{$2005 / 06$} \\
\hline & Booting & Heading & P.M. & Booting & Heading & P.M. \\
\hline & stage & stage & stage & stage & stage & stage \\
\hline \multicolumn{7}{|l|}{ Cropping systems (C): } \\
\hline Pure barley & $0.373 \mathrm{~b}$ & $0.585 \mathrm{~b}$ & $0.852 \mathrm{~b}$ & $0.379 \mathrm{~b}$ & $0.597 \mathrm{~b}$ & $0.864 \mathrm{~b}$ \\
\hline Barley-Vetch & $0.424 \mathrm{a}$ & $0.630 \mathrm{a}$ & $0.890 \mathrm{a}$ & $0.431 \mathrm{a}$ & $0.642 \mathrm{a}$ & $0.901 \mathrm{a}$ \\
\hline Pure vetch & $0.252 \mathrm{c}$ & $0.448 \mathrm{c}$ & $0.688 \mathrm{c}$ & $0.260 \mathrm{c}$ & $0.459 \mathrm{c}$ & $0.698 \mathrm{c}$ \\
\hline F. test $-5 \%$ & * & * & * & * & * & * \\
\hline \multicolumn{7}{|l|}{ P-fertilizer levels (P): } \\
\hline Zero & $0.291 d$ & $0.463 \mathrm{~d}$ & $0.720 \mathrm{~d}$ & $0.296 \mathrm{~d}$ & $0.476 \mathrm{~d}$ & $0.728 \mathrm{~d}$ \\
\hline $7.5 \mathrm{Kg} \mathrm{P}_{2} \mathrm{O}_{5} / \mathrm{fad}$ & $0.328 \mathrm{c}$ & $0.521 \mathrm{c}$ & $0.773 \mathrm{c}$ & $0.335 \mathrm{c}$ & $0.535 \mathrm{c}$ & $0.783 \mathrm{c}$ \\
\hline $15 \mathrm{Kg} \mathrm{P}_{2} \mathrm{O}_{5} / \mathrm{fad}$ & $0.372 \mathrm{~b}$ & $0.592 \mathrm{~b}$ & $0.848 \mathrm{~b}$ & $0.379 \mathrm{~b}$ & $0.603 \mathrm{~b}$ & $0.861 \mathrm{~b}$ \\
\hline $22.5 \mathrm{Kg} \mathrm{P}_{2} \mathrm{O}_{5} / \mathrm{fad}$ & $0.408 \mathrm{a}$ & $0.642 \mathrm{a}$ & $0.899 \mathrm{a}$ & $0.416 \mathrm{a}$ & $0.650 \mathrm{a}$ & $0.911 \mathrm{a}$ \\
\hline F. test $-5 \%$ & * & * & * & * & * & * \\
\hline \multicolumn{7}{|l|}{ Interaction: } \\
\hline $\mathrm{C}$ & 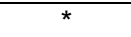 & * & 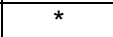 & * & 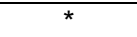 & * \\
\hline
\end{tabular}

*: Significant at 0.05 level of probability.

Meantime, cropping systems interacted significantly with P-fertilizer levels in fresh forage and dry matter yields of both seasons. Results in Table 4 , showed that at all different growth stages, dry matter yield of the tried three cropping systems showed a positive consistent response to P-fertilizer increment up to $22.5 \mathrm{Kg} \mathrm{P}_{2} \mathrm{O}_{5} /$ fad in both seasons. But, under any of the tried P-fertilizer levels, barley-vetch mixture produced higher dry matter yield compared to pure stands of barley and vetch.

Table 4: Dry matter yield ( $t / \mathrm{fad}$ ) as effected by the interaction between cropping systems and P-fertilizer levels at booting, 50\% heading and physiological maturity (P.M) stages in the two seasons

\begin{tabular}{|l|c|c|c|c|c|c|c|}
\hline \multirow{2}{*}{$\begin{array}{l}\text { Cropping } \\
\text { systems }\end{array}$} & $\begin{array}{c}\text { P- } \\
\text { fertilizer } \\
\text { levels }\end{array}$ & $\begin{array}{c}\text { Booting } \\
\text { stage }\end{array}$ & $\begin{array}{c}\text { Heading } \\
\text { stage }\end{array}$ & $\begin{array}{c}\text { P.M. } \\
\text { stage }\end{array}$ & $\begin{array}{c}\text { Booting } \\
\text { stage }\end{array}$ & $\begin{array}{c}\text { Heading } \\
\text { stage }\end{array}$ & $\begin{array}{c}\text { P.M. } \\
\text { stage }\end{array}$ \\
\hline \multirow{3}{*}{$\begin{array}{l}\text { Pure } \\
\text { barley }\end{array}$} & $\mathrm{P}_{0}$ & $0.311 \mathrm{f}$ & $0.503 \mathrm{~g}$ & $0.749 \mathrm{~h}$ & $0.316 \mathrm{f}$ & $0.522 \mathrm{f}$ & $0.769 \mathrm{f}$ \\
\cline { 2 - 8 } & $\mathrm{P}_{1}$ & $0.349 \mathrm{e}$ & $0.546 \mathrm{e}$ & $0.805 \mathrm{f}$ & $0.354 \mathrm{e}$ & $0.554 \mathrm{e}$ & $0.812 \mathrm{e}$ \\
\cline { 2 - 8 } & $\mathrm{P}_{2}$ & $0.396 \mathrm{~d}$ & $0.624 \mathrm{c}$ & $0.903 \mathrm{~d}$ & $0.403 \mathrm{~d}$ & $0.635 \mathrm{c}$ & $0.915 \mathrm{c}$ \\
\cline { 2 - 8 } & $\mathrm{P}_{3}$ & $0.436 \mathrm{c}$ & $0.665 \mathrm{~b}$ & $0.951 \mathrm{~b}$ & $0.443 \mathrm{c}$ & $0.677 \mathrm{~b}$ & $0.961 \mathrm{~b}$ \\
\hline \multirow{3}{*}{$\begin{array}{l}\text { Barley- } \\
\text { vetch }\end{array}$} & $\mathrm{P}_{0}$ & $0.348 \mathrm{e}$ & $0.534 \mathrm{f}$ & $0.798 \mathrm{f}$ & $0.355 \mathrm{e}$ & $0.544 \mathrm{e}$ & $0.809 \mathrm{e}$ \\
\cline { 2 - 8 } & $\mathrm{P}_{1}$ & $0.403 \mathrm{~d}$ & $0.592 \mathrm{~d}$ & $0.852 \mathrm{e}$ & $0.410 \mathrm{~d}$ & $0.614 \mathrm{~d}$ & $0.863 \mathrm{~d}$ \\
\cline { 2 - 8 } & $\mathrm{P}_{2}$ & $0.455 \mathrm{~b}$ & $0.663 \mathrm{~b}$ & $0.928 \mathrm{c}$ & $0.460 \mathrm{~b}$ & $0.675 \mathrm{~b}$ & $0.839 \mathrm{~b}$ \\
\cline { 2 - 8 } $\begin{array}{l}\text { Pure } \\
\text { vetch }\end{array}$ & $\mathrm{P}_{3}$ & $0.491 \mathrm{a}$ & $0.731 \mathrm{a}$ & $0.984 \mathrm{a}$ & $0.498 \mathrm{a}$ & $0.735 \mathrm{a}$ & $0.993 \mathrm{a}$ \\
\cline { 2 - 8 } & $\mathrm{P}_{0}$ & $0.212 \mathrm{j}$ & $0.353 \mathrm{j}$ & $0.612 \mathrm{k}$ & $0.218 \mathrm{i}$ & $0.361 \mathrm{i}$ & $0.607 \mathrm{i}$ \\
\cline { 2 - 8 } & $\mathrm{P}_{1}$ & $0.232 \mathrm{i}$ & $0.425 \mathrm{i}$ & $0.664 \mathrm{j}$ & $0.242 \mathrm{~h}$ & $0.437 \mathrm{~h}$ & $0.676 \mathrm{~h}$ \\
\cline { 2 - 8 } & $\mathrm{P}_{2}$ & $0.265 \mathrm{~h}$ & $0.487 \mathrm{~h}$ & $0.713 \mathrm{i}$ & $0.275 \mathrm{~g}$ & $0.499 \mathrm{~g}$ & $0.730 \mathrm{~g}$ \\
\cline { 2 - 8 } & $\mathrm{P}_{3}$ & $0.298 \mathrm{~g}$ & $0.530 \mathrm{f}$ & $0.764 \mathrm{~g}$ & $0.306 \mathrm{f}$ & $0.539 \mathrm{ef}$ & $0.778 \mathrm{f}$ \\
\hline
\end{tabular}

- Values followed by similar letters are not significantly different at $P<0.05$ by LSD test. $\mathrm{P}_{0}$ : Zero; $\mathrm{P}_{1}$ : $7.5 \mathrm{Kg} \mathrm{P}_{2} \mathrm{O}_{5} /$ fad; $\mathrm{P}_{2}: 15 \mathrm{Kg} \mathrm{P}_{2} \mathrm{O}_{5} /$ fad and $\mathrm{P}_{3}: 22.5 \mathrm{Kg} \mathrm{P}_{2} \mathrm{O}_{5} /$ fad. 
This was the case at different growth stages in both seasons. In other words, these results indicated that mixture which had received $22.5 \mathrm{Kg} \mathrm{P}_{2} \mathrm{O}_{5} / \mathrm{fad}$ could be produced the highest dry matter yield over different growth stages in both seasons, followed by either of pure barley received $22.5 \mathrm{Kg} \mathrm{P}_{2} \mathrm{O}_{5} /$ fad or the mixture received $15 \mathrm{Kg} \mathrm{P}_{2} \mathrm{O}_{5} / \mathrm{fad}$. Where, the latter two treatments produced a comparable dry matter yield in most cases. In this respect, Gurman et al (2006) found that the highest DM yield of vetch was obtained by application of $15 \mathrm{Kg} \mathrm{N}$ and $40 \mathrm{Kg} \mathrm{P} \mathrm{O}_{5} /$ ha.

Protein content $(\%)$ and crude protein yield:

It is evident form results in Table 5 that pure stand of vetch had the highest protein content followed by barley-vetch mixture then pure stand of barley. This was the same over different growth stages in both seasons, but, crude protein content was decreased with the advancing in growth stages. The high dry matter yield potential of sole barley (Table 3) did not compensate the low protein content of barley dry matter, in respect with protein yield i.e. sole vetch produced higher crude protein yield than sole barley as shown in Table 6 . However, the barley-vetch mixture overestimated sole vetch in protein yield of the early two growth stages in both seasons. But, in the latter growth stage of both seasons, crude protein yields of sole vetch and $\mathrm{B}-\mathrm{V}$ mixture were similar. Also, it is observed that $\mathrm{B}-\mathrm{V}$ mixture produced 127.4 and $15.5 \%$ in crude protein yield higher than pure vetch at booting and $50 \%$ heading, respectively in the first season. However, in the second season, these increase percentages were 34.1 and $11.4 \%$ at the two early growth stages in respective order. These results are in harmony with those obtained by Moselhy and El-Hakeem (2001), Ansar et al (2010) and Nadeem et al. (2010).

Results in Tables 4 and 5 showed a gradual significant increase in crude protein content and crude protein yield with each increment in $\mathrm{P}$ fertilizer level up to the higher level tested herein $\left(22.5 \mathrm{Kg} \mathrm{P}_{2} \mathrm{O}_{5} / \mathrm{fad}\right)$. As in dry matter yield, protein yield was also increased with the advancing in growth stage, in spite of the decrease in protein content of the dry matter.

The cropping systems interacted significantly with P-fertilizer levels for crude protein content and crude protein yield as presented in Tables 5 and 6 . As presented in Table 7, the obtained results showed a significant increase for crude protein yield of the three cropping systems with each $\mathrm{P}$-fertilizer increment up to $22.5 \mathrm{Kg} \mathrm{P}_{2} \mathrm{O}_{5} /$ fad. This was the case in different growth stages over both seasons. In opposite with dry matter production under any level of P-fertilizer, crude protein yield of sole vetch was higher than that of sole barley when they harvested at different growth stages in both seasons. Whereas, the mixed cropping system overestimated sole vetch in crude protein yield with some exception especially at latter stage of physiological maturity. Finally, results concluded that mixed cropping system received 22.5 $\mathrm{P}_{2} \mathrm{O}_{5} /$ fad could be maximized crude protein yield at different growth stages. Yet, sole vetch received $22.5 \mathrm{P}_{2} \mathrm{O}_{5} /$ fad could be maximized crude protein yield in the late harvest time at physiological maturity stage. 
Table 5: Effect of cropping systems and P-fertilizer levels on crude protein content (\%) at booting, $50 \%$ heading and physiological maturity (P.M) stages of barley in the two seasons.

\begin{tabular}{|c|c|c|c|c|c|c|}
\hline \multirow[b]{2}{*}{ Treatments } & \multicolumn{3}{|c|}{$2004 / 05$} & \multicolumn{3}{|c|}{$2005 / 06$} \\
\hline & \begin{tabular}{|c|} 
Booting \\
stage
\end{tabular} & $\begin{array}{l}\text { Heading } \\
\text { stage }\end{array}$ & $\begin{array}{l}\text { P.M. } \\
\text { stage }\end{array}$ & $\begin{array}{c}\text { Booting } \\
\text { stage }\end{array}$ & $\begin{array}{c}\text { Heading } \\
\text { stage }\end{array}$ & $\begin{array}{l}\text { P.M. } \\
\text { stage }\end{array}$ \\
\hline \multicolumn{7}{|c|}{\begin{tabular}{|l|l|l|} 
Cropping systems (C): & & \\
\end{tabular}} \\
\hline Pure barley & $3.18 \mathrm{c}$ & $2.84 \mathrm{c}$ & $2.36 \mathrm{c}$ & $3.01 \mathrm{c}$ & $2.58 \mathrm{c}$ & $2.27 \mathrm{c}$ \\
\hline Barley-Vetch & $6.57 \mathrm{~b}$ & $5.90 \mathrm{~b}$ & $4.94 \mathrm{~b}$ & $6.16 \mathrm{~b}$ & $5.33 \mathrm{~b}$ & $4.70 \mathrm{~b}$ \\
\hline Pure vetch & $8.37 \mathrm{a}$ & $7.15 \mathrm{a}$ & $6.46 \mathrm{a}$ & $7.58 \mathrm{a}$ & $6.67 \mathrm{a}$ & $6.12 \mathrm{a}$ \\
\hline F. test- $5 \%$ & * & * & * & ${ }^{\star}$ & * & * \\
\hline \multicolumn{7}{|c|}{ P-fertilizer levels (P): } \\
\hline Zero & $5.21 \mathrm{~d}$ & $4.58 \mathrm{~d}$ & $3.82 \mathrm{~d}$ & $4.88 \mathrm{~d}$ & $4.14 \mathrm{~d}$ & $3.68 \mathrm{~d}$ \\
\hline $7.5 \mathrm{Kg} \mathrm{P}_{2} \mathrm{O}_{5} / \mathrm{fad}$ & $5.71 \mathrm{c}$ & $5.08 \mathrm{c}$ & $4.34 \mathrm{c}$ & $5.32 \mathrm{c}$ & $4.65 \mathrm{c}$ & $4.08 \mathrm{c}$ \\
\hline $15 \mathrm{Kg} \mathrm{P}_{2} \mathrm{O}_{5} / \mathrm{fad}$ & $6.47 \mathrm{~b}$ & $5.62 \mathrm{~b}$ & $4.95 \mathrm{~b}$ & $5.81 \mathrm{~b}$ & $5.11 \mathrm{~b}$ & $4.63 \mathrm{~b}$ \\
\hline $22.5 \mathrm{Kg} \mathrm{P}_{2} \mathrm{O}_{5} / \mathrm{fad}$ & $6.77 \mathrm{a}$ & $5.92 \mathrm{a}$ & $5.24 \mathrm{a}$ & $6.33 \mathrm{a}$ & $5.52 \mathrm{a}$ & $5.07 \mathrm{a}$ \\
\hline F. test- $5 \%$ & * & * & * & * & * & * \\
\hline \multicolumn{7}{|l|}{ Interaction: } \\
\hline$C \times P$ & * & * & * & * & * & * \\
\hline
\end{tabular}

$*$ : Significant at 0.05 level of probability.

Table 6: Effect of cropping systems and P-fertilizer levels on crude protein yield $(\mathrm{Kg} / \mathrm{fad})$ at booting, $50 \%$ heading and physiological maturity (P.M.) stages of barley in both seasons.

\begin{tabular}{|c|c|c|c|c|c|c|}
\hline \multirow[b]{2}{*}{ Treatments } & \multicolumn{3}{|c|}{$2004 / 05$} & \multicolumn{3}{|c|}{$2005 / 06$} \\
\hline & \begin{tabular}{|c|} 
Booting \\
stage
\end{tabular} & $\begin{array}{l}\text { Heading } \\
\text { stage }\end{array}$ & $\begin{array}{l}\text { P.M. } \\
\text { stage }\end{array}$ & $\begin{array}{l}\text { Booting } \\
\text { stage }\end{array}$ & $\begin{array}{l}\text { Heading } \\
\text { Stage }\end{array}$ & $\begin{array}{l}\text { P.M. } \\
\text { stage }\end{array}$ \\
\hline \multicolumn{7}{|c|}{\begin{tabular}{|l|l|} 
Cropping systems (C): & \\
\end{tabular}} \\
\hline Pure barley & $11.94 \mathrm{c}$ & $16.79 \mathrm{c}$ & $20.35 \mathrm{~b}$ & $11.50 \mathrm{c}$ & $15.54 \mathrm{c}$ & $19.84 \mathrm{~b}$ \\
\hline Barley-Vetch & $28.18 \mathrm{a}$ & $37.63 \mathrm{a}$ & $44.44 \mathrm{a}$ & $26.79 \mathrm{a}$ & $34.60 \mathrm{a}$ & $43.18 \mathrm{a}$ \\
\hline Pure vetch & $12.39 \mathrm{~b}$ & $32.59 \mathrm{~b}$ & $44.80 \mathrm{a}$ & $19.99 \mathrm{~b}$ & $31.05 \mathrm{~b}$ & $42.71 \mathrm{a}$ \\
\hline F. test $-5 \%$ & * & * & * & * & * & * \\
\hline \multicolumn{7}{|c|}{ P-fertilizer levels (P): } \\
\hline Zero & $14.63 \mathrm{~d}$ & $20.54 d$ & $26.82 \mathrm{~d}$ & $13.98 d$ & $18.94 \mathrm{~d}$ & $26.08 d$ \\
\hline $7.5 \mathrm{Kg} \mathrm{P}_{2} \mathrm{O}_{5} / \mathrm{fad}$ & $17.94 \mathrm{c}$ & $25.79 \mathrm{c}$ & $32.67 \mathrm{c}$ & $17.26 \mathrm{c}$ & $24.25 \mathrm{c}$ & $31.26 \mathrm{c}$ \\
\hline $15 \mathrm{Kg} \mathrm{P}_{2} \mathrm{O}_{5} / \mathrm{fad}$ & $23.02 \mathrm{~b}$ & $32.49 \mathrm{~b}$ & $40.78 \mathrm{~b}$ & $21.20 \mathrm{~b}$ & $30.03 \mathrm{~b}$ & $38.57 b$ \\
\hline $22.5 \mathrm{Kg} \mathrm{P}_{2} \mathrm{O}_{5} / \mathrm{fad}$ & $26.43 \mathrm{a}$ & $37.19 \mathrm{a}$ & $45.86 \mathrm{a}$ & $25.28 \mathrm{a}$ & $35.03 \mathrm{a}$ & $45.06 \mathrm{a}$ \\
\hline F. test $-5 \%$ & * & * & * & * & * & * \\
\hline \multicolumn{7}{|l|}{ Interaction: } \\
\hline$C \times P$ & n & & & & * & * \\
\hline
\end{tabular}

* Significant at 0.05 level of probability.

Values followed by similar letters are not significantly different at $P<0.05$ by LSD test. 
Table 7: Crude protein yield $(\mathrm{Kg} / \mathrm{fad})$ at three growth stages as effected by the interaction between cropping systems and P-fertilizer levels in the two seasons

\begin{tabular}{|c|c|c|c|c|c|c|c|}
\hline \multirow{2}{*}{\begin{tabular}{|l|} 
Cropping \\
systems
\end{tabular}} & \multirow{2}{*}{\begin{tabular}{|c|} 
P- \\
fertilizer \\
levels \\
\end{tabular}} & \multicolumn{3}{|c|}{ 2004/ 05} & \multicolumn{3}{|c|}{$2005 / 06$} \\
\hline & & $\begin{array}{c}\text { Booting } \\
\text { stage }\end{array}$ & $\begin{array}{c}\text { Heading } \\
\text { stage }\end{array}$ & $\begin{array}{l}\text { P. M } \\
\text { stage }\end{array}$ & $\begin{array}{c}\text { Booting } \\
\text { stage }\end{array}$ & $\begin{array}{c}\text { Heading } \\
\text { stage }\end{array}$ & $\begin{array}{l}\text { P.M. } \\
\text { stage }\end{array}$ \\
\hline \multirow{4}{*}{\begin{tabular}{|l|} 
Pure \\
barley
\end{tabular}} & $\mathrm{P}_{0}$ & $8.98 \mathrm{k}$ & $12.60 \mathrm{j}$ & $14.85 \mathrm{j}$ & $8.35 \mathrm{k}$ & $11.65 \mathrm{k}$ & $15.50 \mathrm{~h}$ \\
\hline & $\mathrm{P}_{1}$ & $10.85 j$ & $15.38 \mathrm{i}$ & $18.13 \mathrm{i}$ & $10.38 \mathrm{j}$ & $13.43 \mathrm{j}$ & $16.75 \mathrm{~h}$ \\
\hline & $\mathrm{P}_{2}$ & $13.23 \mathrm{i}$ & $19.03 \mathrm{~h}$ & $23.15 \mathrm{~h}$ & $12.63 \mathrm{i}$ & $16.75 \mathrm{i}$ & $21.28 \mathrm{~g}$ \\
\hline & $\mathrm{P}_{3}$ & $14.75 \mathrm{~h}$ & $20.18 \mathrm{gh}$ & $25.27 \mathrm{~g}$ & $14.65 \mathrm{~h}$ & $20.33 \mathrm{~h}$ & $25.85 \mathrm{f}$ \\
\hline \multirow{4}{*}{$\begin{array}{l}\text { Barley- } \\
\text { vetch } \\
\text { Mixture }\end{array}$} & $\mathrm{P}_{0}$ & $20.15 f$ & $27.70 \mathrm{f}$ & $32.65 \mathrm{f}$ & $19.40 \mathrm{f}$ & $25.00 \mathrm{~g}$ & $31.98 \mathrm{e}$ \\
\hline & $\mathrm{P}_{1}$ & $24.73 \mathrm{~d}$ & $32.60 \mathrm{~d}$ & $38.18 \mathrm{e}$ & $24.18 \mathrm{~d}$ & $31.45 \mathrm{e}$ & $38.50 \mathrm{~d}$ \\
\hline & $\mathrm{P}_{2}$ & $31.83 \mathrm{~b}$ & $41.60 \mathrm{~b}$ & $50.43 b$ & $29.28 \mathrm{~b}$ & $37.95 \mathrm{c}$ & $45.73 \mathrm{c}$ \\
\hline & $\mathrm{P}_{3}$ & $36.03 \mathrm{a}$ & $48.63 \mathrm{a}$ & $56.53 \mathrm{a}$ & $34.33 \mathrm{a}$ & $44.00 \mathrm{a}$ & $54.63 \mathrm{a}$ \\
\hline \multirow{4}{*}{$\begin{array}{l}\text { Pure } \\
\text { vetch }\end{array}$} & $\mathrm{P}_{0}$ & $14.75 \mathrm{~h}$ & $21.33 \mathrm{~g}$ & $32.95 \mathrm{f}$ & \begin{tabular}{|l|l|}
$14.18 \mathrm{~h}$ \\
\end{tabular} & $20.18 \mathrm{~h}$ & $30.78 \mathrm{e}$ \\
\hline & $\mathrm{P}_{1}$ & $18.25 \mathrm{~g}$ & $29.40 \mathrm{e}$ & $41.70 \mathrm{~d}$ & \begin{tabular}{|l}
$17.23 \mathrm{~g}$ \\
\end{tabular} & $27.88 \mathrm{f}$ & $38.53 \mathrm{~d}$ \\
\hline & $\mathrm{P}_{2}$ & $24.00 \mathrm{e}$ & $36.85 \mathrm{c}$ & $48.78 \mathrm{c}$ & $21.70 \mathrm{e}$ & $35.38 \mathrm{~d}$ & $48.70 \mathrm{~b}$ \\
\hline & $\mathrm{P}_{3}$ & $28.55 \mathrm{c}$ & $42.77 \mathrm{~b}$ & $55.78 \mathrm{a}$ & $26.85 \mathrm{c}$ & $40.78 \mathrm{~b}$ & $54.70 \mathrm{a}$ \\
\hline
\end{tabular}

\section{Grain and straw yields:}

As shown in Table 8, results of the two seasons cleared that the mixed cropping system outyielded pure barley in grain and straw yields, while, pure vetch recorded the lowest values of both these yields. In this respect, these results are in harmony with those obtained by Moselhy and ElHakeem (2001).

Typically as in forage yields, results in Table 8 also exhibited significant positive response to application of P-fertilizer up to $22.5 \mathrm{Kg} \mathrm{P}_{2} \mathrm{O}_{5} /$ fad for grain and straw yields. This was similar in both seasons.

Table 8: Effect of cropping systems and P-fertilizer levels on grain and straw yields ( $t /$ fad) during the two seasons.

\begin{tabular}{|c|c|c|c|c|}
\hline \multirow[b]{2}{*}{ Treatments } & \multicolumn{2}{|c|}{$2004 / 05$} & \multicolumn{2}{|c|}{$2005 / 06$} \\
\hline & $\begin{array}{l}\text { Grain } \\
\text { yield }\end{array}$ & $\begin{array}{l}\text { Straw } \\
\text { yield }\end{array}$ & $\begin{array}{l}\text { Grain } \\
\text { yield }\end{array}$ & $\begin{array}{l}\text { Straw } \\
\text { yield }\end{array}$ \\
\hline \multicolumn{5}{|l|}{ Cropping systems (C ): } \\
\hline Pure barley & $0.623 \mathrm{~b}$ & $1.172 \mathrm{~b}$ & $0.703 \mathrm{~b}$ & $1.176 \mathrm{~b}$ \\
\hline Barley-Vetch & $0.748 \mathrm{a}$ & $1.494 \mathrm{a}$ & $0.810 \mathrm{a}$ & $1.522 \mathrm{a}$ \\
\hline Pure vetch & $0.214 \mathrm{c}$ & $0.861 \mathrm{c}$ & $0.245 \mathrm{c}$ & $0.899 \mathrm{c}$ \\
\hline F. test- $5 \%$ & * & * & * & * \\
\hline \multicolumn{5}{|l|}{ P-fertilizer levels (P): } \\
\hline Zero & $0.365 \mathrm{~d}$ & $0.867 \mathrm{~d}$ & $0.425 \mathrm{~d}$ & $0.877 \mathrm{~d}$ \\
\hline $7.5 \mathrm{Kg} \mathrm{P}_{2} \mathrm{O}_{5} / \mathrm{fad}$ & $0.481 \mathrm{c}$ & $1.029 \mathrm{c}$ & $0.532 \mathrm{c}$ & $1.046 \mathrm{c}$ \\
\hline $15 \mathrm{Kg} \mathrm{P}_{2} \mathrm{O}_{5} / \mathrm{fad}$ & $0.598 \mathrm{~b}$ & $1.325 \mathrm{~b}$ & $0.667 \mathrm{~b}$ & $1.362 \mathrm{~b}$ \\
\hline $22.5 \mathrm{Kg} \mathrm{P}_{2} \mathrm{O}_{5} / \mathrm{fad}$ & $0.669 \mathrm{a}$ & $1.483 \mathrm{a}$ & $0.718 \mathrm{a}$ & $1.511 \mathrm{a}$ \\
\hline F. test $-5 \%$ & * & * & * & * \\
\hline \multicolumn{5}{|l|}{ Interaction: } \\
\hline $\mathrm{C} \times \mathrm{P}$ & * & * & * & * \\
\hline
\end{tabular}

${ }^{*}$ Significant at 0.05 level of probability. 
Results in Table 9 show the significant interaction effect between the studied two factors on grain and straw yields. In both seasons, grain and straw yields for each of the three cropping patterns showed a positive significant response to P-fertilizer increments up to $22.5 \mathrm{Kg} \mathrm{P}_{2} \mathrm{O}_{5} / \mathrm{fad}$. But, under any P-fertilizer level, mixed seeding attained the highest values of both yields followed by pure barley than pure vetch. Accordingly, the highest values of grain and straw yields could be secured when the mixture was fertilized with the higher P-fertilizer level i.e. $22.5 \mathrm{Kg} \mathrm{P}_{2} \mathrm{O}_{5} /$ fad.

Table 9: Grain and straw yields ( $t /$ fad)) as effected by the interaction between cropping systems and P-fertilizer levels in both seasons

\begin{tabular}{|c|c|c|c|c|c|}
\hline \multirow{2}{*}{$\begin{array}{l}\text { Cropping } \\
\text { systems }\end{array}$} & \multirow{2}{*}{$\begin{array}{l}\text { P-fertilizer } \\
\text { levels }\end{array}$} & \multicolumn{2}{|c|}{$2004 / 05$} & \multicolumn{2}{|c|}{$2005 / 06$} \\
\hline & & $\begin{array}{l}\text { Grain } \\
\text { yield }\end{array}$ & $\begin{array}{l}\text { Straw } \\
\text { yield }\end{array}$ & $\begin{array}{l}\text { Grain } \\
\text { yield }\end{array}$ & $\begin{array}{l}\text { Straw } \\
\text { yield }\end{array}$ \\
\hline \multirow{4}{*}{$\begin{array}{l}\text { Pure } \\
\text { barley }\end{array}$} & $\mathrm{P0}$ & $0.421 \mathrm{~g}$ & $0.852 \mathrm{~h}$ & $0.507 \mathrm{e}$ & $0.793 \mathrm{~g}$ \\
\hline & P1 & $0.555 \mathrm{f}$ & $0.987 \mathrm{~g}$ & $0.620 \mathrm{~d}$ & $0.987 \mathrm{f}$ \\
\hline & $\mathrm{P} 2$ & $0.733 \mathrm{~d}$ & $1.374 \mathrm{~d}$ & $0.826 \mathrm{c}$ & $1.405 \mathrm{~d}$ \\
\hline & P3 & $0.781 \mathrm{c}$ & $1.477 \mathrm{c}$ & $0.858 \mathrm{~b}$ & $1.519 \mathrm{c}$ \\
\hline \multirow{4}{*}{$\begin{array}{l}\text { Barley-Vetch } \\
\text { Mixture }\end{array}$} & $\mathrm{P0}$ & $0.552 \mathrm{f}$ & $1.145 \mathrm{f}$ & $0.624 \mathrm{~d}$ & $1.192 \mathrm{e}$ \\
\hline & P1 & $0.719 \mathrm{e}$ & $1.392 \mathrm{~d}$ & $0.789 \mathrm{c}$ & $1.411 \mathrm{~d}$ \\
\hline & P2 & $0.833 \mathrm{~b}$ & $1.665 \mathrm{~b}$ & $0.886 \mathrm{~b}$ & $1.699 \mathrm{~b}$ \\
\hline & P3 & $0.890 \mathrm{a}$ & $1.777 \mathrm{a}$ & $0.939 \mathrm{a}$ & $1.788 \mathrm{a}$ \\
\hline \multirow{4}{*}{$\begin{array}{l}\text { Pure } \\
\text { vetch }\end{array}$} & P0 & $0.123 \mathrm{k}$ & $0.603 \mathrm{j}$ & $0.145 \mathrm{i}$ & $0.646 \mathrm{~h}$ \\
\hline & P1 & $0.168 \mathrm{j}$ & $0.707 \mathrm{i}$ & $0.188 \mathrm{~h}$ & $0.739 \mathrm{~g}$ \\
\hline & $\mathrm{P} 2$ & $0.229 \mathrm{i}$ & $0.937 \mathrm{~g}$ & $0.289 \mathrm{~g}$ & $0.983 \mathrm{f}$ \\
\hline & P3 & $0.336 \mathrm{~h}$ & $1.197 \mathrm{e}$ & $0.358 \mathrm{f}$ & $1.227 \mathrm{e}$ \\
\hline
\end{tabular}

Values followed by similar letters are not significantly different at $\mathbf{P}<0.05$ by LSD test. $P_{0}$ : Zero; $P_{1}: 7.5 \mathrm{Kg} \mathrm{P}_{2} \mathrm{O}_{5} /$ fad; $\mathrm{P}_{2}: 15 \mathrm{Kg} \mathrm{P}_{2} \mathrm{O}_{5} /$ fad and $\mathrm{P}_{3}: 22.5 \mathrm{Kg} \mathrm{P}_{2} \mathrm{O}_{5} /$ fad.

\section{Field stubble yield:}

Data in Table 10 show the yield of field stubble at the end of the two seasons for harvesting at the three growth stages and harvest at full maturity of barley as affected by the two studied factors. The obtained data exhibited that barley-vetch mixture recorded the highest field stubble yield followed by pure barley then pure vetch. This was the same in both seasons after harvest at different growth stages and harvest at full maturity. Also, in both seasons, field stubble yield showed a gradual significant positive response to $\mathrm{P}$ fertilizer increment up to $22.5 \mathrm{Kg} \mathrm{P}_{2} \mathrm{O}_{5} / \mathrm{fad}$ at different growth stages. Meantime, it is seen that field stubble yield was decreased with the advancing in growth stages until the physiological maturity stage. The interactions between cropping systems and P-fertilizer levels were not significant for forage harvest at booting, $50 \%$ heading and physiological maturity stages, but, this interaction was significant for harvest at full maturity of barley. 
Table 10: Effect of cropping systems and P-fertilizer levels on field stubble yield at booting, $50 \%$ heading, physiological maturity (P.M.) and full maturity (F.M.) stages of barley during two seasons

\begin{tabular}{|c|c|c|c|c|c|c|c|c|}
\hline \multirow[b]{2}{*}{ Treatments } & \multicolumn{4}{|c|}{$2004 / 05$} & \multicolumn{4}{|c|}{$2005 / 06$} \\
\hline & $\begin{array}{c}\text { Booting } \\
\text { stage }\end{array}$ & $\begin{array}{l}\text { Heading } \\
\text { stage }\end{array}$ & $\begin{array}{l}\text { P.M. } \\
\text { Stage }\end{array}$ & $\begin{array}{l}\text { F. M. } \\
\text { stage }\end{array}$ & $\begin{array}{c}\text { Booting } \\
\text { stage }\end{array}$ & $\begin{array}{c}\text { Heading } \\
\text { stage }\end{array}$ & $\begin{array}{l}\text { P.M. } \\
\text { Stage }\end{array}$ & $\begin{array}{c}\text { F M. } \\
\text { stage }\end{array}$ \\
\hline \multicolumn{9}{|c|}{ Cropping systems (C): } \\
\hline Pure barley & $148.2 \mathrm{~b}$ & $119.2 \mathrm{~b}$ & $105.8 \mathrm{~b}$ & $106.0 \mathrm{~b}$ & $148.9 \mathrm{~b}$ & $125.5 \mathrm{~b}$ & $115.8 \mathrm{~b}$ & $116.0 \mathrm{~b}$ \\
\hline Barley-Vetch & $155.7 \mathrm{a}$ & $130.1 \mathrm{a}$ & $111.4 \mathrm{a}$ & $111.0 \mathrm{a}$ & $159.3 \mathrm{a}$ & $129.9 \mathrm{a}$ & $119.4 \mathrm{a}$ & $119.0 \mathrm{a}$ \\
\hline Pure vetch & $130.1 \mathrm{c}$ & $108.7 \mathrm{c}$ & $92.7 \mathrm{c}$ & $93.0 \mathrm{c}$ & $135.1 \mathrm{c}$ & $115.4 \mathrm{c}$ & $107.2 \mathrm{c}$ & $107.0 \mathrm{c}$ \\
\hline F. test- $5 \%$ & 末 & * & * & * & * & * & * & 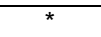 \\
\hline \multicolumn{9}{|c|}{ P-fertilizer levels (P) } \\
\hline Zero & $127.6 \mathrm{~d}$ & $101.2 \mathrm{~d}$ & $78.7 \mathrm{~d}$ & $79.0 \mathrm{~b}$ & $132.0 \mathrm{~d}$ & $108.0 \mathrm{~d}$ & $97.2 \mathrm{~d}$ & $97.0 \mathrm{~d}$ \\
\hline $7.5 \mathrm{Kg} \mathrm{P}_{2} \mathrm{O}_{5} / \mathrm{fad}$ & $136.5 \mathrm{c}$ & $112.6 \mathrm{c}$ & $97.5 \mathrm{c}$ & $98.0 \mathrm{C}$ & $139.8 \mathrm{c}$ & $118.8 \mathrm{c}$ & $110.3 \mathrm{c}$ & $110.0 \mathrm{c}$ \\
\hline $15 \mathrm{Kg} \mathrm{P}_{2} \mathrm{O}_{5} / \mathrm{fad}$ & $152.6 \mathrm{~b}$ & $126.1 \mathrm{~b}$ & $111.7 \mathrm{~b}$ & $112.0 \mathrm{~b}$ & $154.7 \mathrm{~b}$ & $129.4 \mathrm{~b}$ & $120.5 \mathrm{~b}$ & $120.0 \mathrm{~b}$ \\
\hline $22.5 \mathrm{Kg} \mathrm{P}_{2} \mathrm{O}_{5} / \mathrm{fad}$ & $161.9 \mathrm{a}$ & $136.3 \mathrm{a}$ & $125.3 \mathrm{a}$ & $125.0 \mathrm{a}$ & $164.5 \mathrm{a}$ & $138.3 \mathrm{a}$ & $128.7 \mathrm{a}$ & $129.0 \mathrm{a}$ \\
\hline F. test- $5 \%$ & * & ${ }^{*}$ & $x$ & $x$ & * & * & * & \\
\hline \multicolumn{9}{|l|}{ Interaction: } \\
\hline $\mathrm{C} \times \mathrm{P}$ & N.S & N.S & N.S & * & N.S & N.S & N.S & * \\
\hline
\end{tabular}

${ }^{*}$ Significant at 0.05 level of probability. N.S : Not significant at 0.05 level of portability.

\section{Crude protein yield at full maturity:}

As shown in Table 11, barley -vetch mixture produced the highest crude protein yields for grain, straw and field stubble and their total compared to pure stands of barley and vetch in both seasons. Meanwhile, pure barley overestimated pure vetch in grain protein yield of the two seasons, as well as, the total protein yield only in the first season.

Table 11: Effect of cropping systems and P-fertilizer levels on crude protein yields of grain, straw, field stubble and their total $(\mathrm{Kg} / \mathrm{fad})$ during the two seasons.

\begin{tabular}{|c|l|c|c|c|c|c|c|c|}
\hline \multirow{2}{*}{ Treatments } & \multicolumn{3}{|c|}{$2004 / 05$} & \multicolumn{3}{|c|}{$2005 / 06$} \\
\cline { 2 - 6 } & Grain & Straw & $\begin{array}{c}\text { Field } \\
\text { stubble }\end{array}$ & Total & Grain & Straw & $\begin{array}{c}\text { Field } \\
\text { stubble }\end{array}$ & Total \\
\hline Cropping systems (C):
\end{tabular}

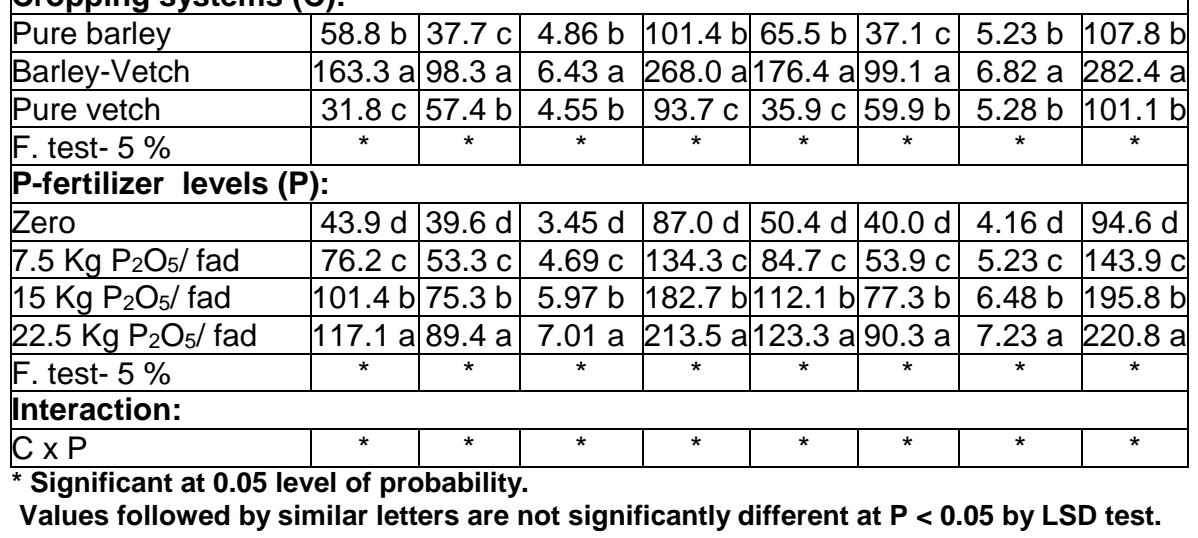


The opposite was true in straw protein yield in comparison between the pure stands of the two crops in favor of pure vetch over the two seasons. However, the pure stands of barley and vetch were at par in protein yield of field stubble in both seasons, as well as, the total crude protein yield only during the second season. Also, it is evident that B-V mixture gave higher total crude protein yield more than pure barley by 164.3 and $162.0 \%$ in the first and second seasons, respectively and higher more than pure vetch by 186.0 and $179.3 \%$ in respective order.

In both seasons, crude protein yields of grain, straw, field stubble and their total were increased consistently with each increment of $P$-fertilizer level up to the higher level $\left(22.5 \mathrm{Kg} \mathrm{P} \mathrm{P}_{5} / \mathrm{fad}\right)$. Though, cropping systems interacted significantly with P-fertilizer levels for protein yields of grain, straw, field stubble and their total in both seasons. Data of this interaction were excluded since they did not afford new information to those of the main effects.

\section{Water use efficiency (WUE):}

Results in Table 12 pointed the superiority of barley-vetch mixture in water use efficiency by grain and SFUs yields over pure stands of barley and vetch. Whereas, pure vetch was gave the lowest values of WUE for grain and SFUs production. This was typically the same in the two seasons. Also, the obtained data showed that B-V mixture was more efficiently in water-use for grain and SFUs production than mono-cropping of barley and vetch, where WUE for grain production was increased by 20.2 and $15.1 \%$ over sole barley and by 249.7 and $230.3 \%$ over sole vetch in the first and second seasons, respectively. However, WUE for SFUs production due to application of mixed cropping system increased by 4.6 and $4.1 \%$ over mono-cropping of barley and by 28.3 and $26.8 \%$ over mono-cropping of vetch in the first and second seasons, in the same respective order.

Table 12: Effect of cropping systems and P-fertilizer levels on water use efficiency (WUE) for grain and SFUs production $(\mathrm{Kg} / \mathrm{mm})$ during the two seasons.

\begin{tabular}{|c|c|c|c|c|}
\hline \multirow[b]{2}{*}{ Treatments } & \multicolumn{2}{|c|}{$2004 / 05$} & \multicolumn{2}{|c|}{$2005 / 06$} \\
\hline & $\begin{array}{l}\text { WUE of } \\
\text { grain }\end{array}$ & $\begin{array}{l}\text { WUE of } \\
\text { SFUs }\end{array}$ & $\begin{array}{l}\text { WUE of } \\
\text { grain }\end{array}$ & $\begin{array}{l}\text { WUE of } \\
\text { SFUs }\end{array}$ \\
\hline \multicolumn{5}{|l|}{ Cropping systems (C): } \\
\hline Pure barley & $5.50 \mathrm{~b}$ & $8.46 \mathrm{~b}$ & $5.02 \mathrm{~b}$ & $6.99 \mathrm{~b}$ \\
\hline Barley-Vetch & $6.61 \mathrm{a}$ & $8.85 \mathrm{a}$ & $5.78 \mathrm{a}$ & $7.28 \mathrm{a}$ \\
\hline Pure vetch & $1.89 \mathrm{c}$ & $6.90 \mathrm{c}$ & $1.75 \mathrm{c}$ & $5.74 \mathrm{c}$ \\
\hline F. test- $5 \%$ & * & * & * & * \\
\hline \multicolumn{5}{|l|}{ P-fertilizer levels (P): } \\
\hline Zero & $3.23 \mathrm{~d}$ & $7.05 \mathrm{~d}$ & $3.03 \mathrm{~d}$ & $5.89 \mathrm{~d}$ \\
\hline $7.5 \mathrm{Kg} \mathrm{P}_{2} \mathrm{O}_{5} / \mathrm{fad}$ & $4.25 \mathrm{c}$ & $7.69 \mathrm{c}$ & $3.80 \mathrm{c}$ & $6.38 \mathrm{c}$ \\
\hline $15 \mathrm{Kg} \mathrm{P}_{2} \mathrm{O}_{5} / \mathrm{fad}$ & $5.29 \mathrm{~b}$ & $8.48 \mathrm{~b}$ & $4.76 \mathrm{~b}$ & $7.00 \mathrm{~b}$ \\
\hline $22.5 \mathrm{Kg} \mathrm{P}_{2} \mathrm{O}_{5} / \mathrm{fad}$ & $5.91 \mathrm{a}$ & $9.05 \mathrm{a}$ & $5.13 \mathrm{a}$ & $7.42 \mathrm{a}$ \\
\hline F. test- $5 \%$ & * & * & * & * \\
\hline \multicolumn{5}{|l|}{ Interaction: } \\
\hline $\mathrm{C} \times \mathrm{P}$ & * & * & * & * \\
\hline
\end{tabular}

${ }^{*}$ Significant at 0.05 level of probability. 
In both seasons, there were a gradual significant increase in WUE for grain and SFUs production with P-fertilizer increments. This could be explained through the favorable role of $\mathrm{P}$-fertilization on root growth. Application of $22.5 \mathrm{Kg} \mathrm{P}_{2} \mathrm{O}_{5} /$ fad increased WUE by 31.6 and $35.0 \%$ for grain production and by 17.7 and $16.3 \%$ for SFUs production due to increasing $\mathrm{P}$ fertilizer levels from 7.5 to $22.5 \mathrm{Kg} \mathrm{P}_{2} \mathrm{O}_{5} /$ fad in the first and second seasons, respectively.

Data of the significant interaction between the two factors on WUE by grain and SFUs in both seasons were excluded, since they did not add valuable information to those of main effects.

\section{Land equivalent ratio (LER):}

The obtained results cleared that B-V mixture had a great advantages over sole seeding in dry matter yield and crude protein yield at different growth stages in both seasons (Table 13), as well as, in grain yield and total crude protein yield at full maturity stage during the two seasons (Table 14).

Table 13: Land equivalent ratio (LER) of barley (LERb) and vetch (LERv) for dry matter yield and crude protein yield at different growth stages in the two seasons

\begin{tabular}{|l|c|c|c|c|c|c|}
\hline \multicolumn{1}{|c|}{$\begin{array}{c}\text { Geason } \\
\text { Growth } \\
\text { stage }\end{array}$} & $\begin{array}{c}\text { Booting } \\
\text { stage }\end{array}$ & $\begin{array}{c}\text { Heading } \\
\text { stage }\end{array}$ & $\begin{array}{c}\text { P.M. } \\
\text { stage }\end{array}$ & $\begin{array}{c}\text { Booting } \\
\text { stage }\end{array}$ & $\begin{array}{c}\text { Heading } \\
\text { stage }\end{array}$ & $\begin{array}{c}\text { P.M. } \\
\text { stage }\end{array}$ \\
\hline Dry matter yield: & & & & & & \\
\hline LER b & 0.764 & 0.7 & 0.73 & 0.768 & 0.712 & 0.723 \\
\hline LER v & 0.552 & 0.491 & 0.39 & 0.538 & 0.473 & 0.395 \\
\hline Total LER & $\mathbf{1 . 3 1 6}$ & $\mathbf{1 . 1 9 1}$ & $\mathbf{1 . 1 2}$ & $\mathbf{1 . 3 0 6}$ & $\mathbf{1 . 1 8 5}$ & $\mathbf{1 . 1 1 8}$ \\
\hline Crude protein yield: & & & & & & \\
\hline LERb & 1.35 & 1.32 & 1.29 & 1.31 & 1.31 & 1.23 \\
\hline LERv & 0.97 & 0.47 & 0.41 & 0.58 & 0.46 & 0.44 \\
\hline Total LER & $\mathbf{2 . 3 2}$ & $\mathbf{1 . 7 9}$ & $\mathbf{1 . 7 0}$ & $\mathbf{2 . 2 6}$ & $\mathbf{1 . 7 7}$ & $\mathbf{1 . 6 7}$ \\
\hline
\end{tabular}

Table 14: Land equivalent ratio (LER) of barley (LERb) and vetch (LERv) for grain yield and crude protein yield at harvest of full maturity stage during the two seasons.

\begin{tabular}{|l|c|c|c|c|}
\hline \multicolumn{1}{|c|}{ Season } & \multicolumn{2}{|c|}{$\mathbf{2 0 0 4 / 0 5}$} & \multicolumn{2}{c|}{$\mathbf{2 0 0 5 / 0 6}$} \\
\hline \multirow{2}{*}{ Traits } & $\begin{array}{c}\text { Grain } \\
\text { Yield } \\
\text { (t/fad) }\end{array}$ & $\begin{array}{c}\text { Total Protein } \\
\text { yield } \\
\text { (Kg/fad) }\end{array}$ & $\begin{array}{c}\text { Grain } \\
\text { yield } \\
\text { (t/fad) }\end{array}$ & $\begin{array}{c}\text { Total Protein } \\
\text { yield } \\
\text { (Kg/fad) }\end{array}$ \\
\hline Barley in mixture & 0.525 & 162.5 & 0.529 & 163.1 \\
\cline { 2 - 5 } Vetch in mixture & 0.223 & 105.5 & 0.281 & 119.3 \\
\hline LER b & 0.843 & 1.60 & 0.752 & 1.52 \\
\cline { 2 - 5 } LER v & 1.040 & 1.13 & 1.147 & 1.18 \\
\hline Total LER & $\mathbf{1 . 8 8 3}$ & $\mathbf{2 . 7 3}$ & $\mathbf{1 . 8 9 9}$ & $\mathbf{2 . 7 0}$ \\
\hline
\end{tabular}

On dry matter yield basis, the advantages in land use were decreased with the advancing in growth stages. Where there was a cooperation relationship between the two mixed components when they harvested at 
booting stage but, a complementary relationship was found in the latter two growth stages. Since, land equivalent ratio for barley (LERb) and land equivalent ratio for vetch (LERv) values in dry matter of the early harvest at booting stage exceeded their sown proportion in mixed seeding rate and this was also the same for LERv but, not for LERb in dry matter yield of the latter harvest at physiological maturity stage. However, at different growth stages in both seasons, the advantages in crude protein yield was higher than that of dry matter yield, but, it was also decrease with the advancing in growth stages. Nevertheless, there were mutual cooperation relationships between the two associated components in crude protein yield of the different growth stages in both seasons. Where, LER value of the mixed two components in this trait exceeded their sown proportion percentages. Similar cooperation relationships were also found in grain and total crude protein yields at full maturity in both seasons (Table 14). Finally, depending on the complementary and cooperative relationships recorded here, it can be stated that mixing of barley with vetch under such conditions is benefit in view of water use efficiencies and yields as well. Hence, it must be recommended as an important agricultural practice under dry weather conditions. These results are in harmony with those obtained by Offori and Stem (1987) and Kurdali et al (1996).

\section{Partial budget analysis:}

As shown in Table (15), total Scandinavian feed units (TSFUs) in terms of total sum of SFUs of grains, straw and field stubble after harvest at full maturity stage was increased by increasing P-fertilizer levels up to $22.5 \mathrm{Kg}$ $\mathrm{P}_{2} \mathrm{O}_{5} /$ fad in each applied cropping system treatment in both seasons. Also, $\mathrm{B}-\mathrm{V}$ mixture produced the highest TSFUs/ fad compared to mono-cropping of both crops. The highest values of gross return (GR) and net return (NR) for total forage products as SFUs/ fad were obtained from barley-vetch mixture when applying the higher level of P-fertilizer $\left(22.5 \mathrm{Kg} \mathrm{P}_{2} \mathrm{O}_{5} / \mathrm{fad}\right)$, while, the lowest values of GR and NR were recorded by mono-cropping of vetch particularly, without application of P-fertilizer in both seasons. In this respect, values of GR follow those for total SFUs in both seasons. The superiority of barley-vetch mixture in GR and NR might be due to the advantages of mixed cropping system with higher LER value and higher productivity of total SFUs/ fad compared to mono-cropping systems for barley and vetch with application the same higher level of P-fertilizer tried herein. This was clearly true in both seasons. However, the highest values of average rate of return (ARR) were recorded by mixed cropping system for two associated crops when fertilized by $15 \mathrm{Kg} \mathrm{P}_{2} \mathrm{O}_{5} /$ fad followed by mono-cropping of barley with the same level of P-fertilizer but, the lowest values were recoded by mono-cropping of vetch under any level of P-fertilize. The same trend was clearly true in both seasons. This was due to the raise in variable costs of vetch seeds and lower SFUs production.

Since, the budget analysis did not take the vital role of crude protein yield in considration, the higher crude protein yield account for B-V mixture, as it is preferable than pure stand of barley. The latter findings are in concurrence with those observed by Papastylianou (1997). Generally, the obtained data of economic analysis indicted that barley-vetch mixture was 
economically than pure stands of both crops particularly, when it fertilized by moderate P-fertilizer level of $15 \mathrm{Kg} \mathrm{P}_{2} \mathrm{O}_{5} /$ fad and could be recommended under rainfed conditions of such area of this study. These findings are in harmony with those obtained by Moselhy and El-Hakeem (2001).

Table 15: Partial budget analysis of total Scandinavian feed units (TSFUs) as affected by cropping systems and P-fertilizer levels during the two seasons of the study.

\begin{tabular}{|c|c|c|c|c|c|c|}
\hline \begin{tabular}{|c} 
Cropping \\
systems
\end{tabular} & $\begin{array}{c}\mathbf{P} \\
\text { levels }\end{array}$ & $\begin{array}{c}\text { TSFUs } \\
\text { (SFU/fad) }\end{array}$ & $\begin{array}{c}\mathrm{GR}^{1} \\
\text { (LE/fad) }\end{array}$ & $\begin{array}{c}\text { TC }^{2} \\
\text { (LE/fad) }\end{array}$ & $\begin{array}{c}N^{3} \\
\text { (LE/fad) }\end{array}$ & $\begin{array}{l}\text { ARR }^{4} \\
\text { (LE) }\end{array}$ \\
\hline \multirow{5}{*}{\begin{tabular}{|l} 
Pure \\
barley
\end{tabular}} & & \multicolumn{5}{|c|}{$2005 / 2006$} \\
\hline & $\mathrm{P}_{0}$ & 883.3 & 1192.5 & 200 & 992.5 & 4.96 \\
\hline & $P_{1}$ & 1098.4 & 1482.8 & 225 & 1257.8 & 5.59 \\
\hline & $\mathrm{P}_{2}$ & 1467.9 & 1981.7 & 250 & 1731.7 & 6.93 \\
\hline & $\mathrm{P}_{3}$ & 1574.2 & 2125.1 & 275 & 1850.1 & 6.73 \\
\hline \multirow{4}{*}{$\begin{array}{l}\text { Barley- } \\
\text { Vetch } \\
\text { Mixture }\end{array}$} & $P_{0}$ & 1155.0 & 1559.2 & 235 & 1324.2 & 5.63 \\
\hline & $P_{1}$ & 1452.1 & 1960.3 & 260 & 1700.3 & 6.54 \\
\hline & $\mathrm{P}_{2}$ & 1701.3 & 2296.7 & 280 & 2016.7 & 7.20 \\
\hline & $P_{3}$ & 1821.7 & 2459.2 & 310 & 2149.2 & 6.93 \\
\hline \multirow{4}{*}{$\begin{array}{l}\text { Pure } \\
\text { vetch }\end{array}$} & $P_{0}$ & 463.9 & 626.2 & 295 & 331.2 & 1.12 \\
\hline & $P_{1}$ & 572.7 & 773.1 & 320 & 453.1 & 1.42 \\
\hline & $\mathrm{P}_{2}$ & 750.2 & 1012.7 & 345 & 667.7 & 1.94 \\
\hline & $P_{3}$ & 990.1 & 1336.6 & 370 & 966.6 & 2.61 \\
\hline \multirow{5}{*}{$\begin{array}{l}\text { Pure } \\
\text { barley }\end{array}$} & & \multicolumn{5}{|c|}{$2006 / 2007$} \\
\hline & $\mathrm{P}_{0}$ & 960.4 & 1488.5 & 200 & 1288.5 & 6.44 \\
\hline & $P_{1}$ & 1175.0 & 1821.2 & 225 & 1596.2 & 7.09 \\
\hline & $\mathrm{P}_{2}$ & 1581.6 & 2451.4 & 250 & 2201.4 & 8.81 \\
\hline & $P_{3}$ & 1674.1 & 2594.8 & 275 & 2319.8 & 8.44 \\
\hline \multirow{4}{*}{\begin{tabular}{|l} 
Barley- \\
Vetch \\
Mixture
\end{tabular}} & $P_{0}$ & 1262.1 & 1956.3 & 235 & 1721.3 & 7.32 \\
\hline & $P_{1}$ & 1538.6 & 2384.8 & 260 & 2124.8 & 8.17 \\
\hline & $P_{2}$ & 1776.1 & 2752.9 & 280 & 2472.9 & 8.83 \\
\hline & $P_{3}$ & 1879.3 & 2912.9 & 310 & 2602.9 & 8.40 \\
\hline \multirow{4}{*}{$\begin{array}{l}\begin{array}{l}\text { Pure } \\
\text { vetch }\end{array} \\
\text { a }\end{array}$} & $P_{0}$ & 529.2 & 820.3 & 295 & 525.3 & 1.78 \\
\hline & $P_{1}$ & 626.1 & 970.4 & 320 & 650.4 & 2.03 \\
\hline & $\mathrm{P}_{2}$ & 843.5 & 1307.3 & 345 & 962.3 & 2.79 \\
\hline & $P_{3}$ & 1028.1 & 1593.5 & 370 & 1223.5 & 3.31 \\
\hline
\end{tabular}

1): GR: gross return; 2): TC: total costs; 3): NR: net return and 4): ARR: average rate of return $\mathrm{P}_{0}$ : Zero; $\mathrm{P}_{1}$ : $7.5 \mathrm{Kg} \mathrm{P} \mathrm{O}_{5} /$ fad; $\mathrm{P}_{2}: 15 \mathrm{Kg} \mathrm{P}_{2} \mathrm{O}_{5} /$ fad and $\mathrm{P}_{3}: 22.5 \mathrm{Kg} \mathrm{P}_{2} \mathrm{O}_{5} /$ fad.

\section{REFERENCES}

Ansar M.; Z. I. Ahmed; M. A. Maik; M. Nadeem; A. Majeed and B. A. Rischkowsky (2010). Forage yield and quality potential of winter cereal-vetch mixtures under rainfed conditions. Emir. J. Food Agric. 22 (1): 25-36.

Fujita K.; S. Ogata; K. Matsumoto; T. Masuda; G. K. Ofosu-Budu and K. Kuwata (1990). Nitrogen transfer and dry matter production in soybean and sorghum mixed cropping system at different population densities. Soil Sci. Plant Nutr. 36: 233-241.

Gurman Z. A.; M. Qamar; S. Shafeeq and M. Sh. Zahid (2006). Effect of phosphorus fertilizer application on fodder and grain yield of vetch under rainfed conditions of Pothowar region. Pak. J. Agric. Sci., 43 (1-2): 17-20. 
Izaurralde R. C.; W. B. McGill and N. G. Juma (1992). Nitrogen fixation efficiency, interspecies $\mathrm{N}$ transfer, and root growth in barley field pea intercrop on a black chernozemic soil. Biol. Fertil. Soils, 13: 11-16.

Karadag Y. and U. Buyukburc (2001). Research on the effects of different phosphorus doses on root, nodule and plant growth in some vetch species. Turkish Journal of Agriculture and Forestry, 25 (6): 359-368.

Kurdali F.; N. E. Sharabi and A. Arslan (1996). Rainfed vetch-barley mixed cropping in the Syrian semi-arid conditions. Plant and Soil J., 183: 137-148.

Le Houerou, H. N. (1986). Salt tolerant plants of economic value in the Mediterranean basin. Reclamation and Re-vegetation Research, 5:319-341.

Le Houerou, H. N. (1994). Drought-tolerant and water-efficient fodder shrubs (DTFS), their role as a "drought-tolerance" in the agriculture development of arid and semi-arid zones in southern Africa. Report to the Water Research Commission No. KV 65/94 WRC, Pretoria, South Africa.

Lithourgidis A. S.; I. B. Vasilakoglou; K. V. Dhima; C. A. Dordas and M. D. Yiakoulaki (2006). Forage yield and quality of common vetch mixtures with oat and triticale in two seeding ratios. Field Crops Res., 99: 106-113.

Mead R. and R. W. Willey (1980). The concept of a "Land Equivalent Ratio" and advantages in yield from intercropping. Exp. Agric. 16, 217-228.

Mohsenabadi Gh. R.; M. R. Jahansooz; M. R. Chaichi; H. Rahimian Mashhadi; A. M. Liaghat and Gh. R. Savaghebi (2008). Evaluation of barley-vetch intercrop at different nitrogen rates. J. Agric. Sci. Technol., 10: 23-31.

Moselhy, N. M. M. and M. S. El-Hakeem (2001). Yield potentiality of barley-vetch mixtures under rainfed conditions in Northwest coast of Egypt. Zagazig J. Agric. Res., 28 (5): 763-778.

MRMP. (2002). Matrouh Resource Management Project (MRMP) Documents. 870, -IDA Credit 2504-EGT. PP: 65-73.

Nadeem M.; M. Ansar; A. Anwar; A. Hussain and S. Khan (2010). Performance of winter cereal-legumes fodder mixtures and their pure stand at different growth stages under rainfed conditions of Pothowar. J. Agric. Res., 48 (2):181-192.

Offori F. and W. R. Stem (1987). Cereal-legume intercropping systems. Adv. Agron., 41: 41-90.

Papastylianou I. and S. K. A. Danso (1989). Effect of nitrogen fertilization and cropping system of the reference crop on estimation of $\mathrm{N}_{2}$-fixation by vetch using ${ }^{15} \mathrm{~N}$ methodology. Plant and Soil J. 114: 223-227.

Papastylianou I. and S. K. A. Danso (1991). Nitrogen fixation and transfer in vetch and vetch-oats mixtures. Soil Biol. Biochem., 23: 447-452.

Perrin, R. K.; D. L. Winkelmann; E. R. Moscardi and J. R. Anderson (1983). An Economics Training Manual, CIMMYT, Information Bulletin 27, $5^{\text {th }}$ Printing. D.F., $54 \mathrm{pp}$.

Qamar I. A.; J.D.H. Keatinge; Noor Muhammad; A. Aly and M. A. Khan (1999). Introduction and management of vetch-barley forage mixtures in the rainfed areas of Pakistan, 1. Forage yield. Australian J. of Agric. Res., 50: 1-9.

Snedecor, G. W. and Cochran, W. G. (1990). " Statistical Methods". 8 the ed. lowa State Univ. Press, Ames, lowa, USA. 
Turk, M. A. and A. M. Tawaha (2001). Common vetch (Vicia sativa L.) productivity as influenced by rate and method of phosphate fertilization in a Mediterranean environment. Agric. Mediterranean 131 (3-4): 108-111.

إسـتجابة مخلـوط الثـعير والبقيـا لمستويات التسـميد الفوسـفاتى تحت ظروف الزراعـة

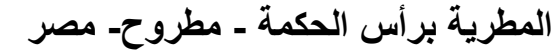

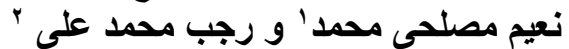

' شعبة البيئة وزراعات المناطق الجافة ـ مركز بحوث بحث الصحراء، ومدير مركز التنمية المستدامة

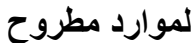

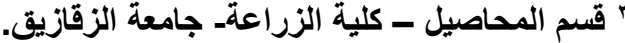

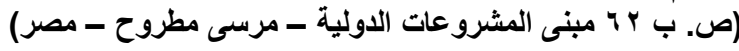

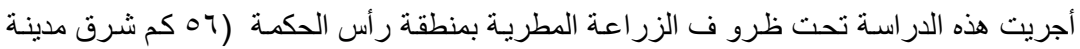

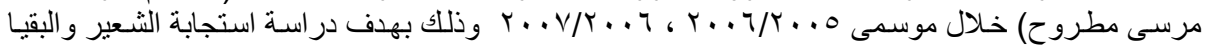

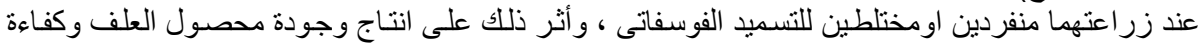

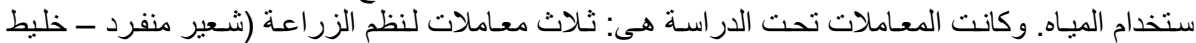

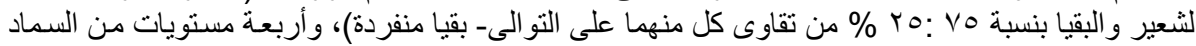

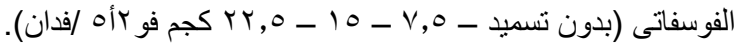

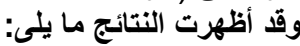

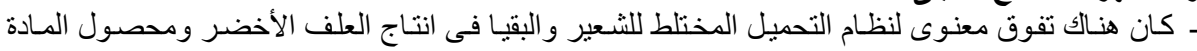

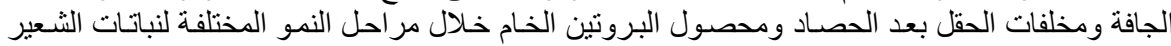

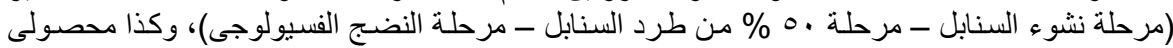

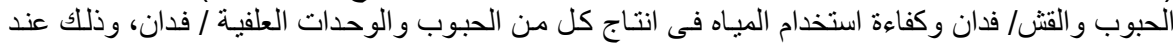

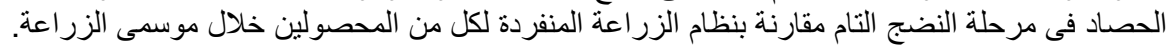

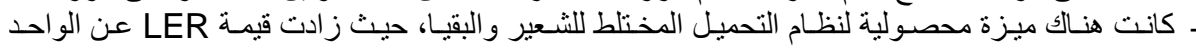

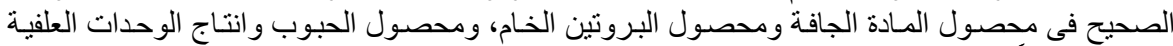

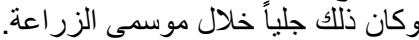

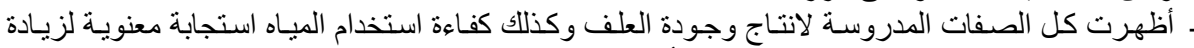

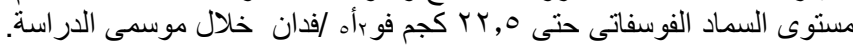

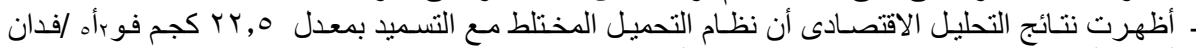

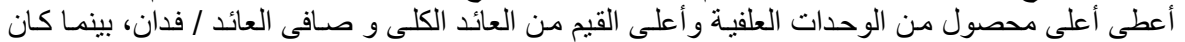

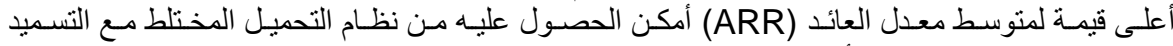

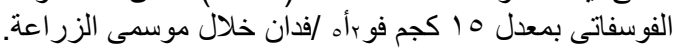

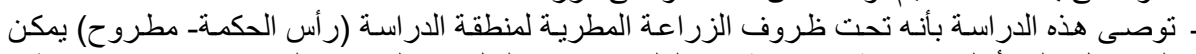

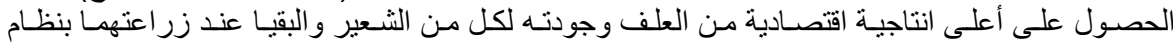

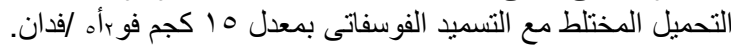

كلية الزراعة - جامعة المنصورة

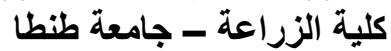

قام بتحكيم البحث

أ. أد / على السعيد اليحث شريف

أ.د /السيد حامد الصعيدي 